1

\title{
Embedded Fluorescence Lifetime Determination for High-throughput, Low-photon-number Applications
}

\author{
Tobias Lieske • Wilfried Uhring - Norbert Dumas • Jérémie Léonard • \\ Dietmar Fey
}

Received: date / Accepted: date

\begin{abstract}
Time-resolved fluorescence (TRF) analysis is considered to be among the primary research tools in biochemistry and biophysics. One application of this method is the investigation of biomolecular interactions with promising applications for biosensing. For the latter context, time-correlated single photon counting (TCSPC) is the most sensitive, hence preferred implementation of TRF. However, high throughput applications are presently limited by the maximum achievable photon acquisition rate, and even more by the data processing rate. The latter rate is actually
\end{abstract}

This work was financially supported by the Research Training Group 1773 "Heterogeneous Image Systems", funded by the German Research Foundation (DFG).

\section{Tobias Lieske}

Friedrich-Alexander-University Erlangen-Nürnberg (FAU), Department of Computer Science, Chair of Computer Architecture, Martensstraße 3, 91058 Erlangen, Germany +49 91318520208

E-mail: Tobias.Lieske@fau.de

Wilfried Uhring

ICube Laboratory (UMR 7357), University of Strasbourg and CNRS, France

E-mail: Wilfried.Uhring@icube.unistra.fr

Norbert Dumas

ICube Laboratory (UMR 7357), University of Strasbourg and CNRS, France

E-mail: Norbert.Dumas@icube.unistra.fr

Jérémie Léonard

Université de Strasbourg, CNRS, Institut de Physique et Chimie des Matériaux de Strasbourg and Labex NIE, UMR 7504, F-67000 Strasbourg, France

E-mail: Jeremie.Leonard@ipcms.unistra.fr

Dietmar Fey

Friedrich-Alexander-University Erlangen-Nürnberg (FAU), Department of Computer Science, Chair of Computer Architecture, Martensstraße 3, 91058 Erlangen, Germany

E-mail: Dietmar.Fey@fau.de limited by the computational complexity to estimate accurately the fluorescence lifetime from TCSPC data. Here we propose a solution that would enable the implementation of TRF detection for fluorescence-activated droplet sorting (FADS), a particularly high throughput, microfluidic-based technology.

Most fluorescence lifetime algorithms require a large number of detected photons for an accurate lifetime computation. This paper presents an implementation based on a maximum likelihood estimator (MLE), enabling high precision estimation with a limited number of detected photons, significantly reducing the total measurement time. This speedup rapidly increases the input data rate. As a result, off-the-shelf embedded products cannot handle the data rates produced by current TCSPC units that are used to measure the fluorescence. Therefore, a configurable real-time capable hardware architecture is implemented on a fieldprogrammable gate array (FPGA) that can handle the data rates of future TCSPC units, rendering high throughput droplet sorting with microfluidics possible. The presented hardware architecture is validated with experimental input data and produces high precision results.

Keywords FPGA · time-resolved fluorescence · fluorescence lifetime · microfluidics · embedded signal processing

\section{Introduction}

Fluorescence describes the emission of light from an electronically excited state. The lifetime $\tau$ is the average time between its excitation and the emission of a photon. Fluorescence spectroscopy and time-resolved fluorescence analysis methods are among the primary re- 
search tools in biochemistry and biophysics, but are not limited to these fields. The main biotechnological applications include flow cytometry, medical diagnostics, DNA sequencing, forensics and genetic analysis [11]. A typical example that benefits from increased throughput is fluorescence-activated droplet sorting (FADS) [3] that is used for drug discovery [20].

Fluorescence spectroscopy measures the average emission intensity, but results underlie many influences such as turbidity, sample concentration, geometry of the sample and chromophore bleaching. Furthermore, this analysis method cannot capture important information, such as fluorescence emitted by one fluorophore masking other fluorophores due to spectral overlap as well as interactions of the fluorophore with the substrate or other macromolecules [11].

Time-resolved fluorescence analysis methods on the other hand offer more information and are more robust, but require sophisticated optics and electronics due to the short time scale of fluorescence processes, which typically have emission rates of $10^{8} s^{-1}$ [11]. The analysis is based on the fluorescence lifetime, which has no dependency on emission intensity $[6,18]$ and is independent of the probe concentration as long as the probe emission is well above the background signal [11]. The fluorescence lifetime enables a distinct classification.

Microfluidics is a technology that allows the processing of small amounts of fluids $\left(10^{-9}\right.$ to $10^{-18}$ liters), utilizing channels with diameters of tens to hundreds of micrometers. This analysis technique offers various benefits such as carrying out separations and detections with high resolution and sensitivity, low cost and short times for analysis [24]. In order to combine the robustness of time-resolved fluorescence analysis with high throughput microfluidics, a sufficiently fast data processing is required to form a reliable high throughput sensing method. A particularly challenging example is microdroplet or cell sorting. The proposed experiment setup is illustrated in Fig. 1. A low cost high repetition rate picosecond laser diode generator is focused with lens $\mathrm{L}_{1}$ on the microdroplets in the channel, causing fluorescence. The emitted photons (dotted lines) pass the dichroic mirror unimpeded, are collected by lens $\mathrm{L}_{2}$ and then hit the single photon avalanche diode (SPAD). A time to digital converter (TDC) measures the time between the excitation and the emission of the photon. Based on this information, a histogram of arrival times can be generated, see Fig. 2. The fluorescence intensity is defined by Eq. 1, where $I_{0}$ is the initial intensity and $\tau$ the fluorescence lifetime [11]. The histogram of arrival times in Fig. 2 reflects the exponential decay character of the fluorescence intensity and can be used to extract the fluorescence lifetime $\tau$. The measured

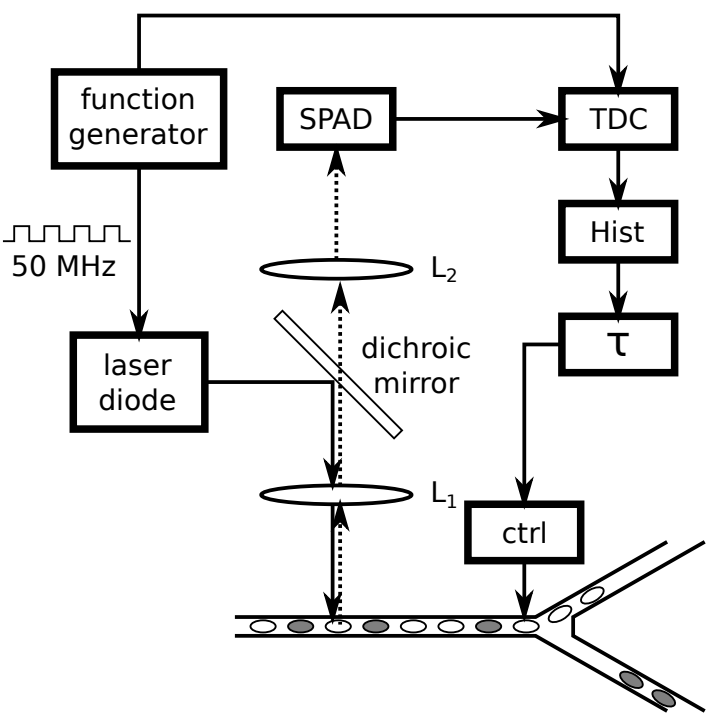

Fig. 1: Schematic setup of a microfluidics sorting system based on time-resolved fluorescence.

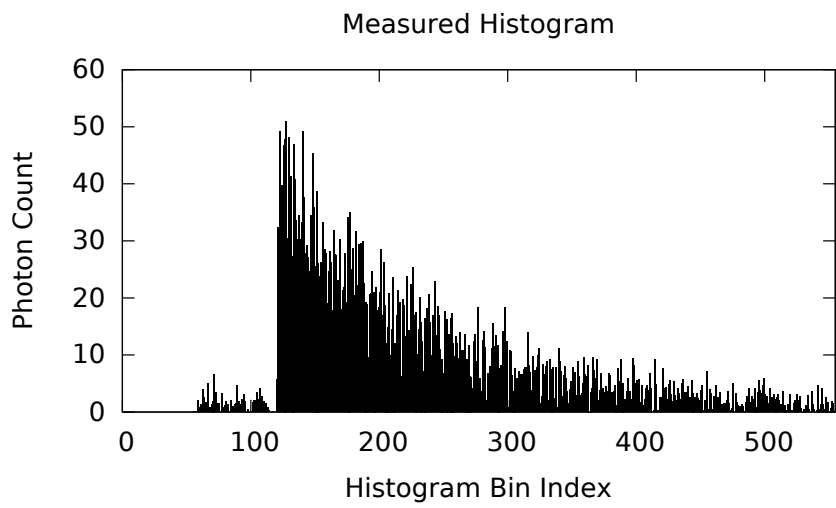

Fig. 2: Histogram of arrival times. Each bin represents a time slot and contains the number of photons detected in that slot.

$I(t)=I_{0} e^{-\frac{t}{\tau}}$

lifetime is then used to separate the classified microdroplets correctly further back in the channel. Due to the flow velocity, the fluorescence lifetime computation has a hard real-time deadline set at $5 \mathrm{~ms}$.

The challenge of this approach is the complexity of the time-resolved fluorescence analysis method. Microfluidics systems have no problem generating a laminar high throughput flow of up to twenty thousand so called microdroplets per second [22]. However, there are currently two problems that limit the actual throughput of such a sorting system. The photon counting rate for current time-correlated single-photon counting (TCSPC) systems is limited by the sample's 
lifetime, the pileup effect and dead-times of electronics, which will all be discussed in Section 3 .

In a previous experimental work, we have investigated how the pile-up effect and the photon shot noise - inherent to single photon detection - may limit the applications of TCSPC in very high throughput conditions [12]. More specifically, we have demonstrated that these limitations do not preclude the use of TCSPC to detect with high sensitivity and good accuracy the fluorescence lifetime in microfluidic droplets of $50 \mathrm{mi}-$ crons diameter circulating at a flow rate exceeding $10^{3}$ droplets per second. Recently, we applied the same experimental concept to demonstrate the feasibility and very good reliability of a biologically-relevant enzymatic activity assay based on fluorescence lifetime detection, in 200-picoliter droplets circulating at 300 droplets per second, by implementing TCSPC [9]. In these previous experimental demonstrations, the data analysis (automatic droplet detection, building and fitting of fluorescence histograms for individual droplets) was done a posteriori, by post-processing the acquired data.

In this paper, we aim at enabling the real-time data processing in such experiments. This would be of central relevance to extend the above proof-of-principle experiments to microfluidic droplet sorting or fluorescence assisted cell sorting (FACS) experiments based on fluorescence lifetime detection by TCSPC. More specifically, we demonstrate the FPGA implementation of a monoexponential decay fit in order to achieve the processing of up to 151 histograms per millisecond, in conditions of low photon number per histogram.

Established methods for fluorescence lifetime computation require large photon counts for precise estimation, resulting in a low throughput. Algorithms based on a maximum likelihood estimator (MLE) deliver reliable high precision results even with low photon counts per histogram. This drastically reduces the required measurement time and in turn increases the possible input data rate. Transferring the data to a powerful workstation consumes too much time and embedded off-the-shelf products cannot handle the resulting data rate. Therefore, a dedicated low latency, high throughput embedded hardware architecture for highly accurate fluorescence lifetime classification based on low signal-to-noise inputs has been developed. This new architecture renders high throughput microfluidic sorting possible with current TCSPC systems. The system has been introduced in [15] and is discussed here in more detail and validated with real experimental input data.

The following section reviews systems and methods for fluorescence lifetime extraction. After that, factors limiting the counting rate of TCSPC electronics for fluorescence measurements are explained in Section 3 and the MLE algorithm is revised in Section 4. Subsequently, the hardware architecture is explained in Section 5 and compared to existing approaches in Section 6 . The paper ends with a validation of the presented hardware architecture with experimental input data in Section 7 and a brief conclusion in Section 8 .

\section{Related Work}

The following review focuses on the fluorescence lifetime extraction from a given histogram of arrival times, as displayed in Fig. 2.

The work presented in [5] shows a complete system similar to Fig. 1. While the TDC and histogram generation is implemented on a FPGA, the fluorescence lifetime is being computed externally on a workstation. Therefore, the histogram data is transferred via UART (Universal Asynchronous Receiver Transmitter). The results mention a delay of a few seconds to measure the fluorescence decay of a substance, which is clearly too slow for the real-time control system required by the sorting experiment proposed in this paper. Another approach using external processing is introduced in [7]. The fluorescence signals are transmitted via TCP/IP (Transmission Control Protocol/Internet Protocol) to a workstation that performs the lifetime computation. The signal acquisition, preprocessing and TCP/IP transmittance time is given as $9.5 \mathrm{~ms}$. This time does not include the actual computation of the lifetime, proving that external computation of the fluorescence lifetime does not meet the given time constraints and an embedded approach is necessary to avoid long data transfer times.

There are several algorithms available that calculate the fluorescence lifetime. These algorithms can be classified into two groups. The first type are model based fitting methods such as Bayesian [19], maximum likelihood [17] or maximum entropy [21], method of moments [8] and least squares deconvolution with Laguerre expansion [16,7]. These fitting methods return precise results, but have a relatively high computational complexity and easily converge to a local minimum. Furthermore, each model is derived from application knowledge such as the number of lifetimes contained in the data and thus the fitting approach is limited to the selected data [25].

The second algorithm class are nonfitting methods. Examples are the rotational invariance technique introduced in [25], the phasor analysis [4], Prony's method [26], the integral equation method [14] and the center of mass method [13,23]. These algorithms offer a greatly reduced computational complexity when 
compared to the fitting based methods. Yet, some approaches are limited to monoexponential decays. An evaluation of these approaches is given in [25]. As will be shown in Section 6.2, the estimation quality of nonfitting methods is subpar and model based fitting methods offer superior results.

However, the main goal is to speed up the sorting process while maintaining the measurement precision. [17] shows that the maximum likelihood estimation (MLE) performs better on low signal-to-noise fluorescence decays than the popular least squares method and a classification of monoexponential decays is possible with very few photons [10]. In addition to that, the computational complexity for fluorescence lifetime estimation using the MLE algorithm is significantly lower than for the least squares algorithm [8]. Therefore, the MLE algorithm has been selected for further analysis, since collecting less photons per droplet results in a shorter measurement time and in a higher experiment throughput.

\section{Counting Rates of TCSPC Electronics}

As already mentioned, the photon counting rate of TCSPC systems is limited by three factors. For one, TCSPC electronics exhibits a dead-time after a photon has been detected. Thus, photons arriving later at the SPAD can be masked by photons arriving early. This effect is called pulse pileup and deforms the recorded histogram in such a way that the measured fluorescence lifetime is reduced. This problem is usually addressed by following the $1 \%$ rule that states the excitation energy of one laser pulse should be so low that only one electron is emitted out of 100 excitation pulses. However, this $1 \%$ rule is being considered as overcautious. Counting rates at $10 \%$ decrease the lifetime by less than $1 \%$ and even a $30 \%$ count rate results only in a modest change [11]. Therefore, the technically maximum pulse repetition rate of the used laser can limit the photon counting rates. On the other hand, the maximum pulse repetition rate in order to determine the lifetime properly depends on the fluorescence decay times of the sample. The time between the pulses should be at least four times the longest lifetime in the sample [11]. Another technical limit is the actual duration of the dead-time of the TCSPC electronics. Effective counting rates further drop by $50 \%$ [11]. Considering an example with a lifetime of $5 \mathrm{~ns}$, a maximum laser repetition rate of $50 \mathrm{MHz}$ is allowed. Targeting a $30 \%$ count rate results in 15 Mcps (counts per second), which can be handled by currently available TCSPC products [2]. As will be shown in Section 6.2, a total photon count of 500 per histogram is enough for precise lifetime extraction with an average error below 0.5 $\%$ using the MLE algorithm. Provided one achieves a sufficiently fast fitting rate, up to 30000 microdroplets could be sorted per second. Our objective is therefore to implement a fitting routine that may process up to several $10^{4}$ histograms per second.

\section{Fluorescence Lifetime Estimation}

The maximum likelihood estimator for monoexponential fluorescence decay signals has been derived in [8] and is given in Eq. 2. With $x=T / \tau, T$ the time step of

$1+\left(e^{x}-1\right)^{-1}-m\left(e^{m x}-1\right)^{-1}=\bar{\nu}$

one histogram bin, $m$ the number of histogram bins, $\bar{\nu}$ as defined in Eq. 3 and $N_{i}$ the number of photons in his-

$\bar{\nu}=\frac{\sum_{i}^{m} i N_{i}}{\sum_{i}^{m} N_{i}}$

togram bin $i$. The term $\bar{\nu}$ can be seen as the histogram bin index with the "average" number of photons. The left-hand side of Eq. 2 is strictly monotone decreasing and admits a unique solution as long as the source is decaying. Therefore, a solution can be easily obtained by applying the Newton-Raphson method, as displayed in Eqs. 4 to 6.

$$
\begin{aligned}
L(x) & =1+\left(e^{x}-1\right)^{-1}-m\left(e^{m x}-1\right)^{-1}-\bar{\nu}=0 \\
x_{i+1} & =x_{i}-\frac{L\left(x_{i}\right)}{L^{\prime}\left(x_{i}\right)} \\
& =x_{i}-\frac{1+\left(e^{x}-1\right)^{-1}-m\left(e^{m x}-1\right)^{-1}-\bar{\nu}}{m^{2} e^{m x}\left(m e^{m x}-1\right)^{-2}-e^{x}\left(e^{x}-1\right)^{-2}}
\end{aligned}
$$

Depending on the measured time window length, the total number of detected photons and the fluorescence lifetime, there is a visible noise floor, which can be seen in Fig. 2. As already mentioned, the photon count for the high throughput sorting application is relatively low. Therefore, higher index photon counts are relatively speaking stronger influenced by this noise floor, as these bins generally have a very low photon count. The noise floor deforms the exponential decay 
and directly influences $\bar{\nu}$ and thus the estimated fluorescence lifetime value $\tau$, see Eq. 3. Therefore, an iterative noise removal process called auto-windowing has been developed to acquire an improved approximation of the fluorescence lifetime. Depending on the estimated fluorescence lifetime, the input histogram is truncated to remove noisy bins and the fluorescence lifetime is recomputed, until a stable result is acquired.

This algorithm has been implemented in $\mathrm{C}$ on an ARM $^{\circledR}{ }^{\circledR}$ Cortex ${ }^{\circledR}$-A53 quad-core processor. Processing one histogram of 1024 bins takes 152.365 us. Assuming no delays caused by concurrent memory accesses, a total of 26.25 histograms per millisecond can be processed with this embedded processor, which is not sufficient to handle the data rate of 30 histograms per millisecond of the numerical example given in Section 3 . However, TCSPC electronics continuously improve enabling higher counting rates. Additionally, measuring a lifetime less than 5 ns allows higher laser repetition rates resulting in even higher counting rates, which in turn cannot be handled by the ARM $^{\circledR}$ Cortex ${ }^{\circledR}$ - A53 quad-core processor.

\section{Hardware Implementation}

Therefore, a dedicated hardware implementation has been developed in VHDL to avoid compute-bound fluorescence measurement systems and maximize the sorting throughput. The presented fluorescence lifetime computation system is displayed in the block diagram in Fig. 3 connected to the histogram and control unit, as shown in the experiment setup in Fig. 1. The input and output interfaces use asynchronous FIFO (first-infirst-out) buffers for clock synchronization. The actual computation system consists of a preprocessing stage and a computation stage. The first part addresses the input data and performs some preprocessing to compute $\bar{\nu}$ for Eq. 6. The second stage implements the Newton-Raphson iteration of the estimator function Eq. 6 to compute the fluorescence lifetime $\tau$. This pipeline-like system structure enables concurrent processing of input data.

\subsection{Preprocessing}

The preprocessing stage is designed as a streaming architecture that accepts each input histogram bin by bin and computes the $\bar{\nu}$ value, which is required to solve Eq. 6. As displayed in Eq. 3, $\bar{\nu}$ depends on the number of histogram bins. However, the histogram size changes when the auto-windowing approach truncates the histogram. Therefore, different $\overline{\nu_{i}}$ values will be required

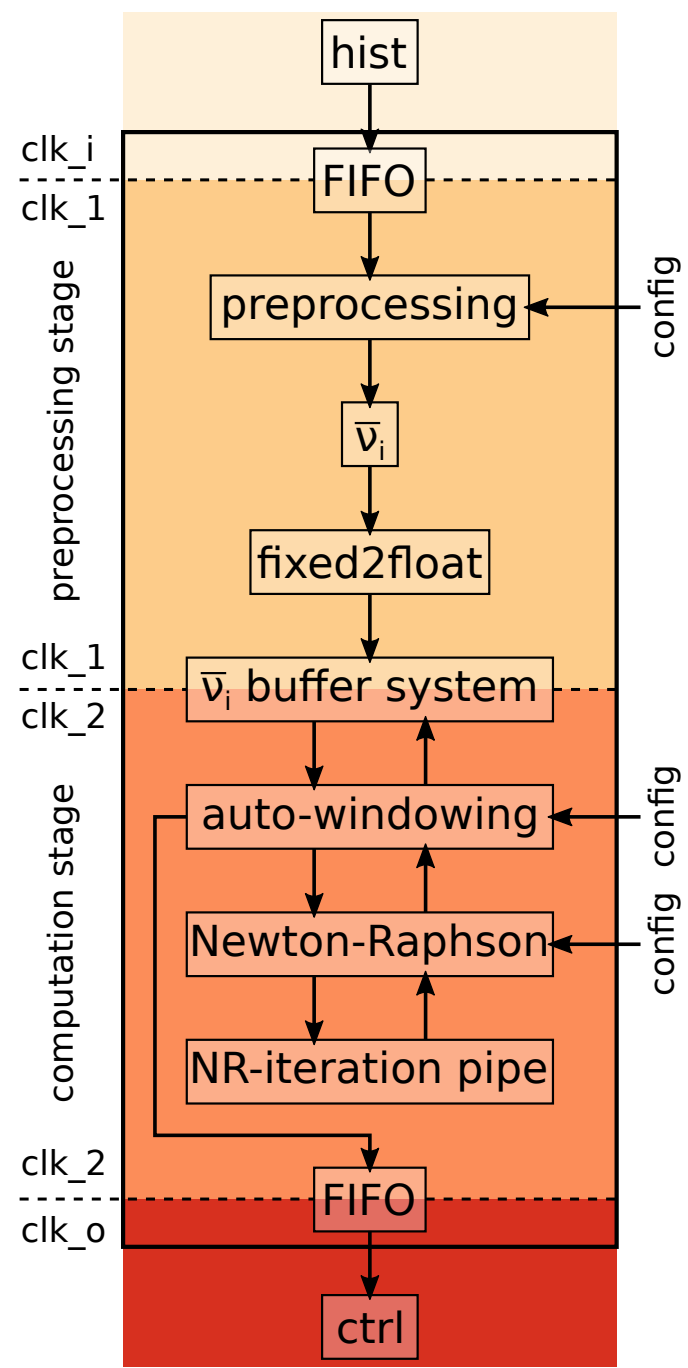

Fig. 3: System overview of the presented fluorescence lifetime computation unit.

to compute the fluorescence lifetime with this iterative approach. In order to avoid any delays by on-demand computations, $\overline{\nu_{i}}$ is computed on the fly for every histogram bin index $i$ and stored in a buffering system.

The computations in this stage are performed on fixed-point data that scales throughout the module depending on the configurable input data width. This approach reduces the hardware resource requirements as opposed to a floating-point based implementation. Furthermore, the critical path of the preprocessing stage is reduced. In combination with the separate clock regions for the preprocessing and computation stages, this increases the maximum input data frequency and therefore the system throughput. The measurements performed in Sections 6 and 7 show that the fixed-point computation does not impair a precise fluorescence life- 
time computation when compared to a floating-point implementation.

The preprocessing module in Fig. 3 is required to extract the actual histogram that represents the exponential decay function from the noisy input provided by the histogram generation module, see Fig. 2. The two main noise components in such a measured histogram are the noise floor caused by background light and spikes in the histogram caused by Poisson noise, which is inherent for photon counting processes [11]. In order to compensate both noise sources, the start of the histogram is computed as the mean index position of the maximum, which is determined within a configurable window at the beginning of the input histogram. In the course of an experiment, temperature changes influence the TDC behavior and can thus gradually shift the beginning of the histogram. Therefore, the mean maximum index is only computed over a dynamically configurable window size of the last histograms to account for this behavior.

While the preprocessing can be implemented in a fully pipelined streaming fashion, both the autowindowing approach and the Newton-Raphson method are iterative algorithms. In order to maximize the throughput, the computed $\overline{\nu_{i}}$ values are stored in a buffer system as single-precision floating point values. The $\overline{\nu_{i}}$ values of different input histograms are stored in separate buffers slots and accessible to the second stage to enable concurrent computations of Eq. 6 .

\subsection{Fluorescence Lifetime Computation}

The computation of the fluorescence lifetime is performed in the computation stage of the system, see Fig. 3. Due to the large dynamic range of the exponential terms in Eq. 6, floating-point values are used instead of fixed point. In order to avoid that the critical path of floating-point arithmetic degrades the performance of the preprocessing stage, separate clocks are used for each stage and clock synchronization is implemented in the $\overline{\nu_{i}}$ buffer system module.

The second stage is fully pipelined, although autowindowing and Newton-Raphson are both iterative algorithms. The $\overline{\nu_{i}}$ buffer system in Fig. 3 signals the auto-windowing module when a new complete set of $\overline{\nu_{i}}$ values is available. The auto-windowing module in turn creates a new computation job and dispatches it to the Newton-Raphson module. A computation job consists of a number of job properties that are listed in Table 1. These are the current iteration indices $j$ and $k$ for the auto-windowing and Newton-Raphson iterations, the current histogram size $m_{j}$, which decreases
Table 1: Computation job properties.

\begin{tabular}{|c|c|}
\hline Job Property & Value \\
\hline \hline AW-Iteration & $j$ \\
\hline NR-Iteration & $k$ \\
\hline hist size & $m_{j}$ \\
\hline $\bar{\nu}$ & $\overline{\nu_{m_{j}}}$ \\
\hline
\end{tabular}

Table 2: Experiment-wide job properties.

\begin{tabular}{|c|c|}
\hline Experiment Property & Value \\
\hline \hline AW-Limit & $j_{\text {limit }}$ \\
\hline NR-Limit & $k_{\text {limit }}$ \\
\hline Time Step & $T$ \\
\hline Initial Lifetime & $\tau_{0}$ \\
\hline
\end{tabular}

with each auto-windowing iteration due to the truncation process, and the current $\bar{\nu}$ value which depends on the current histogram size. Further runtime parameters are set experiment-wide when configuring the computation system. These are listed in Table 2. The iteration limits $j_{\text {limit }}$ and $k_{\text {limit }}$ can be set within the range of statically configured values $J_{\text {limit }}$ and $K_{\text {limit }}$ set at synthesis time. Apart from the histogram data itself, Eq. 4 requires information about the time step $T$ of one histogram bin during the measurement and an initial value $x_{0}=T / \tau_{0}$. Therefore, the time step $T$ and an initial fluorescence lifetime $\tau_{0}$ are part of the configuration as well. Nevertheless, the computed fluorescence lifetime empirically converges within ten Newton-Raphson iterations, regardless of $\tau_{0}$.

With the job properties listed in Tables 1 and 2, the Newton-Raphson module displayed in Fig. 3 computes $k_{\text {limit }}$ iterations of Eq. 6 in order to solve Eq. 4 and get a first estimate of the fluorescence lifetime $\tau$. Once the Newton-Raphson iteration reaches $k_{\text {limit }}$, the computed fluorescence lifetime $\tau$ is passed to the auto-windowing module where the histogram size $m_{j}$ for the next autowindowing iteration is derived and the corresponding $\overline{\nu_{m_{j}}}$ is loaded from the $\overline{\nu_{i}}$ buffer system to update the job state in Table 1.

Figure 3 shows that the Newton-Raphson module controls the NR-iteration pipe block, which is a fully pipelined implementation of Eq. 6. In order to avoid numeric instability, critical parts of the computation are performed in double-precision. By pipelining the $N R$-iteration pipe block, up to 130 fluorescence lifetime computations can be performed in parallel each identified by a tag to ensure unambiguous assignment of input histogram to output lifetime. In reality, the number of parallel computation jobs is bound to the statically configurable number of buffer slots in the $\overline{\nu_{i}}$ buffer system module that is required to synchronize the input histogram stream with the computation per- 
Table 3: Configuration parameters for implementation results in Table 4.

\begin{tabular}{|c|c|c|c|c|}
\hline Data Width & $m_{\max }$ & $J_{\text {limit }}$ & $K_{\text {limit }}$ & \#Buffer \\
\hline 32 & 1024 & 10 & 10 & 16 \\
\hline
\end{tabular}

formance. This means, the fluorescence lifetime computation for preprocessed histogram data stored as $\overline{\nu_{i}}$ values in buffer slot $l$ has to be completed by the computation stage before the preprocessing stage overwrites the $\overline{\nu_{i}}$ values in slot $l$ with data from a new input histogram. Using the input bin data frequency and the worst-case iteration counts $J_{\text {limit }}$ and $K_{\text {limit }}$, the minimum number of buffer slots can be computed easily, as will be shown in Section 6 .

Using this system structure, multiple preprocessing stages can be connected to share one fluorescence lifetime computation stage to save resources.

\section{Evaluation}

6.1 Implementation Results

In order to evaluate the presented fluorescence lifetime computation system, a parameter set for the configuration has to be selected. Table 3 shows an excerpt of the static configuration parameters and their values used for the implementation results in Table 4 obtained by Quartus Prime 15.1.0. The Data Width defines the input bit width of a histogram bin represented as fixed point number. $m_{\max }$ configures buffer sizes and sets the maximum supported histogram size. AW Iter max $_{\text {con- }}$ figures the counter bit width for the auto-windowing iterations. The actual iteration count can be set dynamically between 1 and $A W$ Iter $_{\max }$. The same applies for the Newton-Raphson iteration count that is configured

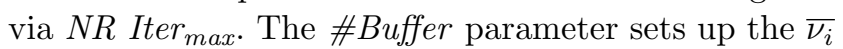
buffer system.

An FPGA-based TDC and histogram generation module is introduced in [5]. Such a module can be implemented along with the here presented fluorescence lifetime computation system on the same FPGA chip, given the unused resources in Table 4. Input histograms are streamed to the preprocessing stage bin by bin sequentially. Thus, the reported maximum clock frequency in Table 4 for the preprocessing stage corresponds to a potential input data stream of 151 histograms per millisecond, each histogram 1024 bins wide. Such a histogram rate may occur when the fluorescence lifetime needs to be measured at several points along the microfluidics channel or when counting rates of TCSPC electronics further improve.
Filling 1 of the 16 buffers in the $\overline{\nu_{i}}$ buffer system takes about 6.59 us (1024 cycles). Assuming a continuous input data stream, the time window to process the data just stored in this buffer is about $98.98 \mathrm{\mu s}$ (15360 cycles), which is the time to fill 15 buffers. Running the computation with the highest iteration count of 10 auto-windowing iterations, each consisting of 10 Newton-Raphson iterations, takes 93.86 us (13840 cycles).

This means that even though an iterative algorithm has been chosen for the fluorescence lifetime computation, the presented architecture is IO bound due to smart parallel processing strategies. The given configuration can easily process 151 histograms per millisecond with a maximum computation delay of about 100,46 us per histogram on a low cost FPGA development board [1]. The throughput and BRAM resource utilization of the system can be scaled down, by reducing the size of the $\overline{\nu_{i}}$ buffer system. In case higher input data rates need to be processed, several preprocessing stages can be connected with some glue logic to one shared computation stage.

\subsection{Quality of Computed Fluorescence Lifetimes}

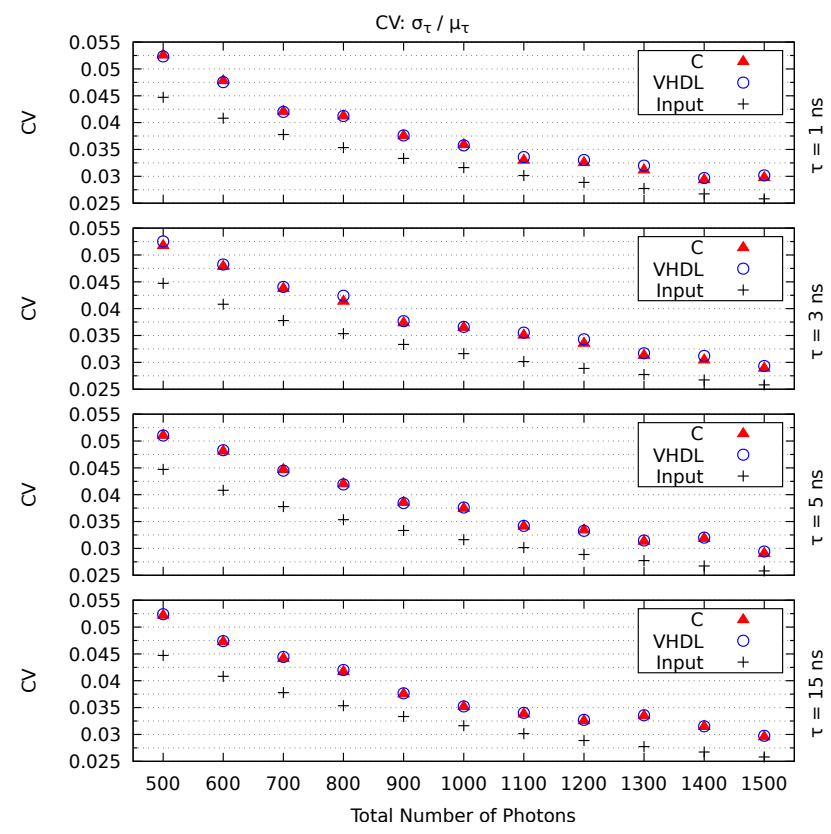

Fig. 4: Coefficient of variation for the $\mathrm{C}$ and VHDL implementation, as well for the input histogram.

In order to test the quality of the computed fluorescence lifetimes, input histograms for four fluorescence 
Table 4: Implementation results on Altera Cyclone V 5CSEMA4U23C6.

\begin{tabular}{|c|c|c|c|c|c|}
\hline$f_{\text {stage_1 }}$ & $f_{\text {stage_2 }}$ & FF & ALM & DSP & BRAM \\
\hline $155.18 \mathrm{MHz}$ & $147.45 \mathrm{MHz}$ & $29891(47 \%)$ & $11937(75 \%)$ & $71(85 \%)$ & $228(84 \%)$ \\
\hline
\end{tabular}

Table 5: Time step and histogram size for each $\tau$ test case.

\begin{tabular}{|c||r|r|r|r|}
\hline$\tau[\mathrm{ns}]$ & 1 & 3 & 5 & 15 \\
\hline$T[\mathrm{ps}]$ & 45 & 90 & 90 & 90 \\
\hline$m$ & 512 & 512 & 512 & 1024 \\
\hline
\end{tabular}

lifetimes $\tau \in\{1 \mathrm{~ns}, 3 \mathrm{~ns}, 5 \mathrm{~ns}, 15 \mathrm{~ns}\}$ have been generated. The time step $T$ and number of histograms bins $m$ have been selected according to Table 5 . Poisson noise has been added to the histograms, since this kind of noise is inherent for photon counting processes and also is the prominent kind of noise for low photon counts [11]. Further, the behavior for low photon counts needs to be analyzed. Therefore, histograms with a different total photon count $N \in\{500,600, \ldots, 1500\}$ have been generated. Due to the Poisson noise, the total number of photons deviates and is not exact for each histogram. For every $\{\tau, N\}$ set, 1000 histograms have been generated and the corresponding lifetime has been computed with the MLE algorithm implemented in $\mathrm{C}$ and by simulating the VHDL design.

Figure 4 lists the coefficient of variation (CV), which is the quotient of the standard deviation to the mean. This ratio is a standardized measure of dispersion. The results show that the noise introduced by the processing is in an acceptable range compared to the input noise ( $\mathrm{CV}$ of Poisson distribution is $1 / \sqrt{N}$ ) and decreases with increasing $N$. Slight variations are caused by the added Poisson noise. Some histograms are noisier than others and some sets include more of such "noisier" histograms. Similarly, $N$ varies from histogram to histogram.

The non fitting algorithms analyzed in [25] are compared by their normalized bias $(\Delta \tau / \tau)$ and the $F$-value, which is the normalized precision defined as $F=\sqrt{N}$. $\sigma_{\tau} / \mu_{\tau}$. An ideal computation has $F=1$, while realistic computations feature $F>1$ and $F \gg 1$ [25]. The histograms used in [25] have $m=1024$ and a time bin width $T=12.5 \mathrm{~ns} / 1024 \approx 12.2 \mathrm{ps}$, which is a much finer temporal resolution than the one used for the test cases in this paper, see Table 5. Unfortunately, $N$ is not given, but the number of photons in the first bin is, which is either 1000 when $\tau$ is being varied or $\tau$ is fixed at $3 \mathrm{~ns}$ and the number of photons in the first bin varies from 100 to 5000 . In order to put these numbers into context, the mean photon count in the first bin where the exponential decay starts is listed in Table 6

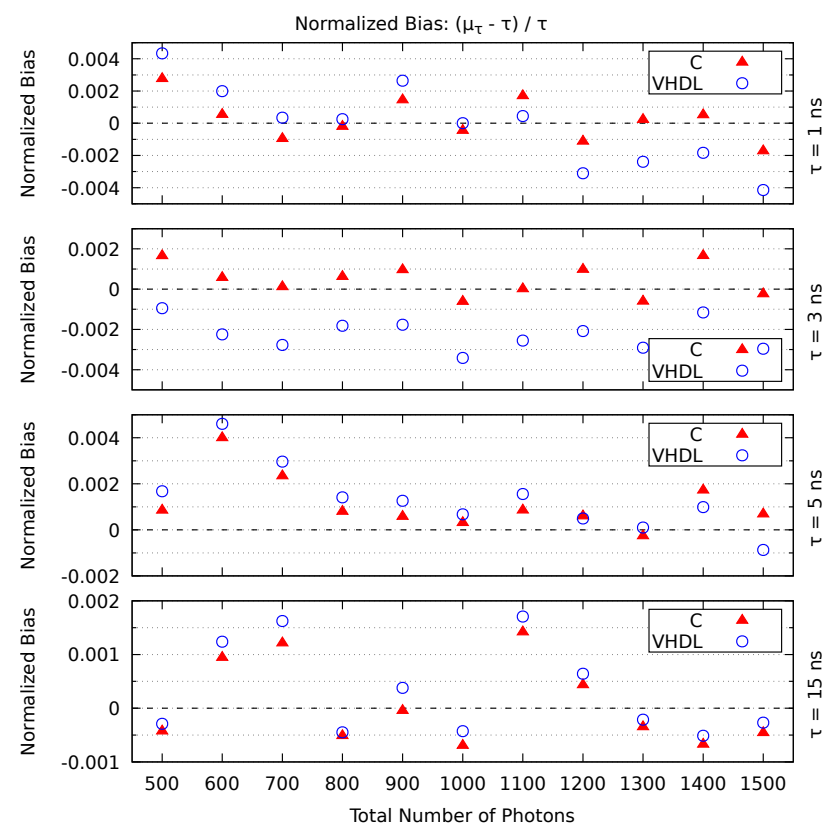

Fig. 5: Normalized bias for $\mathrm{C}$ and VHDL implementation. The optimum is a bias of 0 .

for all test cases used in this paper. It is obvious that the test cases used in this paper feature a drastically lower total photon count $N$, which implies a much shorter measurement time per droplet and thus improves the throughput. Nonetheless, the resulting bias and F-value measurements presented in Figs. 5 and 6 respectively, show that the MLE algorithm returns superior results.

The bias for all measurements plotted in Fig. 5 is clearly below $0.5 \%$, while the bias reported in [25] for $\tau$ $=3 \mathrm{~ns}$ for the integral equation method (IEM) is about $-1.6 \%$ and about $6.3 \%$ for the center of mass method (CMM). Both clearly exceeding the bias of the MLE. Further, the listed phasor analysis features a bias of about $0.8 \%$ ( $\tau=3 \mathrm{~ns}$ ), while the presented fluorescence lifetime estimation via rotational invariance techniques (FLERIT) algorithm is very close to $0 \%$ bias $(\tau=3 \mathrm{~ns})$. Extracting an exact range from the plot is not possible due to the coarse scale. The CMM method is discarded for the further comparison considering its excessive lifetime dependent bias.

Looking at the F-values reported in [25], it is clear that FLERIT, IEM and phasor exhibit a lifetime dependent precision. The authors list optimized regions for the analyzed algorithms, where $F<4$. These re- 
Table 6: Mean photon count in the first bin of the exponential decay.

\begin{tabular}{|r||r|r|r|r|r|r|r|r|r|r|r|}
\hline$\tau[\mathrm{ns}]$ & 500 & 600 & 700 & 800 & 900 & 1000 & 1100 & 1200 & 1300 & 1400 & 1500 \\
\hline \hline 1 & 22.38 & 27.00 & 31.61 & 34.88 & 40.09 & 43.34 & 48.86 & 52.15 & 57.67 & 61.78 & 67.17 \\
\hline 3 & 13.34 & 16.56 & 19.19 & 21.85 & 24.50 & 27.10 & 29.24 & 33.07 & 35.16 & 37.88 & 41.23 \\
\hline 5 & 8.83 & 10.35 & 11.89 & 13.80 & 15.63 & 16.89 & 18.84 & 20.77 & 22.18 & 24.05 & 25.63 \\
\hline 15 & 2.97 & 3.46 & 4.10 & 4.60 & 5.24 & 5.89 & 6.57 & 7.31 & 7.68 & 8.24 & 8.86 \\
\hline
\end{tabular}
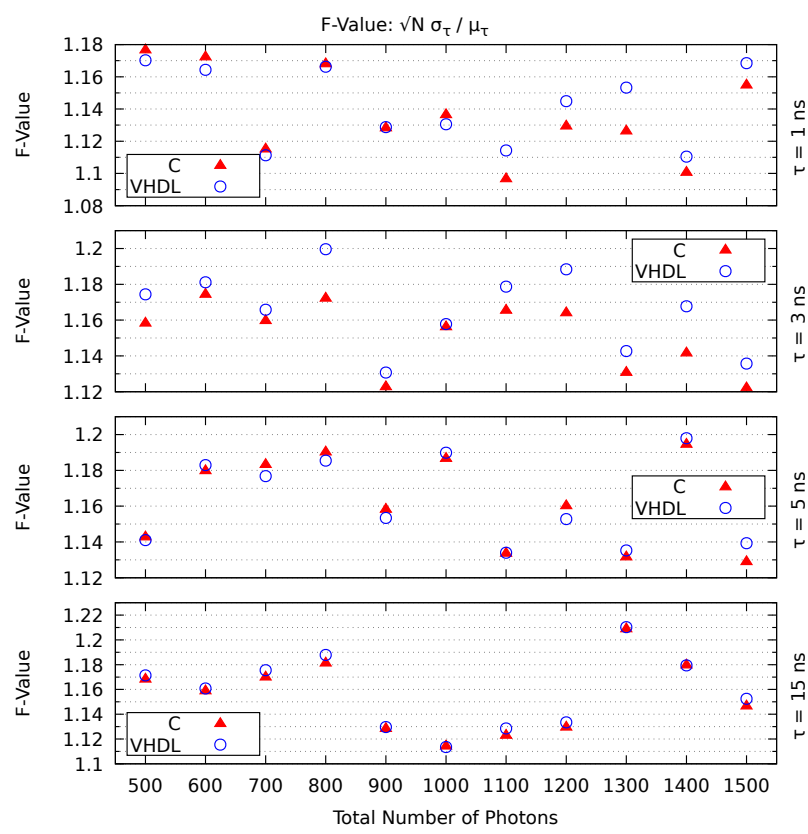

Fig. 6: F-Value for C and VHDL implementation.

gions are from $0.4 \mathrm{~ns}$ to $14 \mathrm{~ns}$ for FLERIT, $0.4 \mathrm{~ns}$ to $8.8 \mathrm{~ns}$ for IEM and $0.4 \mathrm{~ns}$ to $4.23 \mathrm{~ns}$ for phasor. A higher resolution plot for $\tau=3$ ns shows that the F-value for the two best algorithms FLERIT and IEM varies between 1.5 and 2.0 [25]. In contrast to this, the F-value measurements of the MLE algorithm given in Fig. 6 show no dependency on the lifetime and no test case exceeds a F-value of 1.22.

\section{Application to Real Experimental Data}

In order to assess the performances of the above fitting procedure and VHDL implementation with real data, we use experimental data recorded in a proofof-principle experiment demonstrating the feasibility of an enzymatic activity assay based on fluorescence lifetime detection, at very high-throughput in microfluidic droplets [9]. More specifically, in this assay, a fluorescently labeled substrate is used such that if the enzyme is active, the fluorophore is cleaved from the substrate and its fluorescence lifetime increases. Two data sets named 1 and 2 , were acquired by implement-

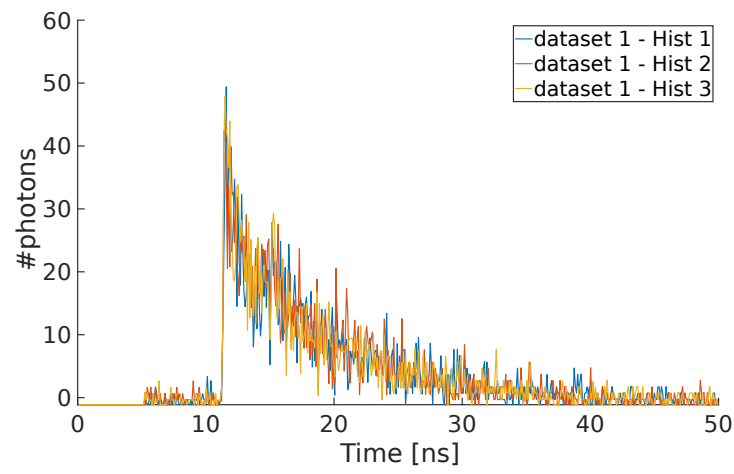

(a) Exemplary histograms from data set 1 .

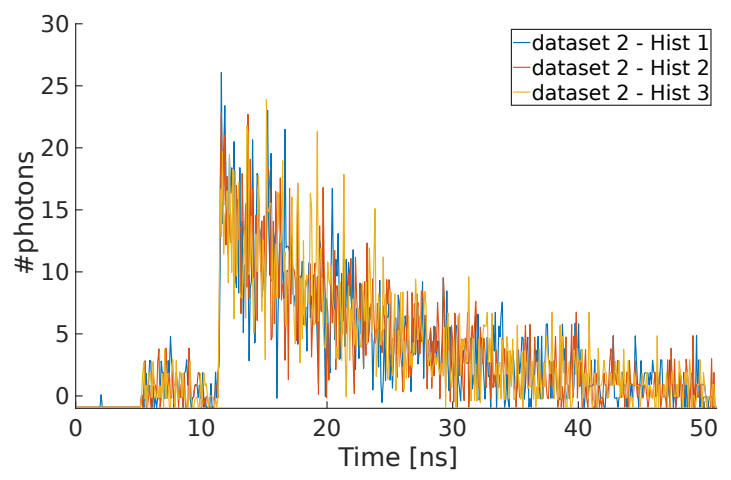

(b) Exemplary histograms from data set 2.

Fig. 7: Exemplary histogram data acquired from the experimental measurement setup in [9] with additional postprocessing to remove the non-zero offset.

ing TCSPC in microfluidic droplets (as in Fig. 1, see also [9]) at a throughput of 300 droplets per second, in conditions where about 2500 single photons were collected per droplet. Two successive experiments where performed to generate data set 1 and 2 in which the droplet contents were the bare substrate (data set 1) or the substrate cleaved by the enzyme (data set 2). A histogram of the time-stamped single photon detection events was constructed for each droplet, by off-line data post-processing. Figures $7 \mathrm{a}$ and $7 \mathrm{~b}$ display exemplary three histograms from data set 1 and data set 2 respectively. Here we present the analysis of the two sets of histograms with the presented VHDL fitting scheme. 
Table 7: Statistical properties for the computation results for data sets 1 and 2 from Fig. 8a and the corresponding reference results from [9].

\begin{tabular}{|c|c|c|c|c||c|c|c|}
\cline { 2 - 8 } \multicolumn{1}{c|}{} & \multicolumn{4}{c||}{ VHDL } & \multicolumn{3}{c|}{ reference } \\
\cline { 2 - 9 } \multicolumn{1}{c|}{} & $\mathrm{u}[\mathrm{ns}]$ & sig $[\mathrm{ns}]$ & $\mathrm{CV}[\%]$ & F-Value & $\mathrm{u}[\mathrm{ns}]$ & sig $[\mathrm{ns}]$ & CV $[\%]$ \\
\hline data set 1 & 8.3 & 0.25 & 3.0 & 1.5 & 6.9 & 0.25 & $\sim 3.6$ \\
\hline data set 2 & 12.2 & 0.47 & 3.8 & 1.8 & 11.4 & 0.42 & $\sim 3.6$ \\
\hline
\end{tabular}

Table 8: Statistical properties for the computation results for data sets 1 and 2 from Fig. 8b and the corresponding reference results from [9].

\begin{tabular}{|c|c|c|c|c||c|c|c|}
\cline { 2 - 8 } \multicolumn{1}{c|}{} & \multicolumn{4}{c||}{ VHDL } & \multicolumn{3}{c|}{ reference } \\
\cline { 2 - 8 } \multicolumn{1}{c|}{} & $\mathrm{u}[\mathrm{ns}]$ & sig $[\mathrm{ns}]$ & $\mathrm{CV}[\%]$ & F-Value & $\mathrm{u}[\mathrm{ns}]$ & sig [ns] & CV [\%] \\
\hline data set 1 & 8.1 & 0.07 & 0.9 & 1.4 & 6.9 & 0.08 & $\sim 1.2$ \\
\hline data set 2 & 12.1 & 0.14 & 1.1 & 1.7 & 11.2 & 0.13 & $\sim 1.2$ \\
\hline
\end{tabular}

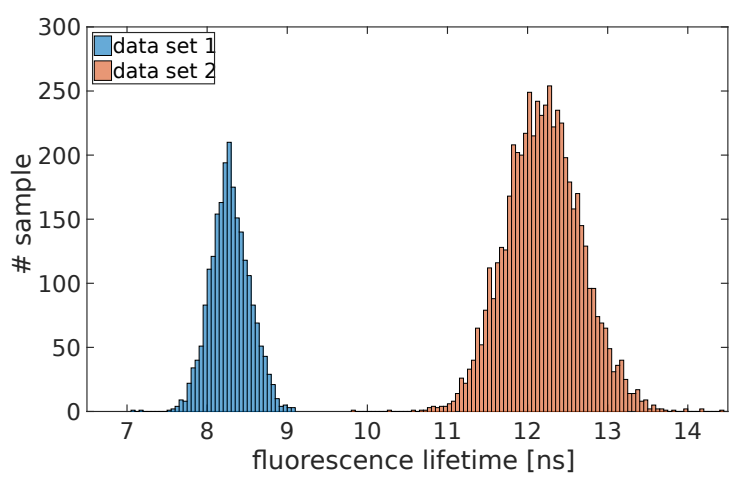

(a) Fluorescence lifetime distribution for data sets 1 and 2.

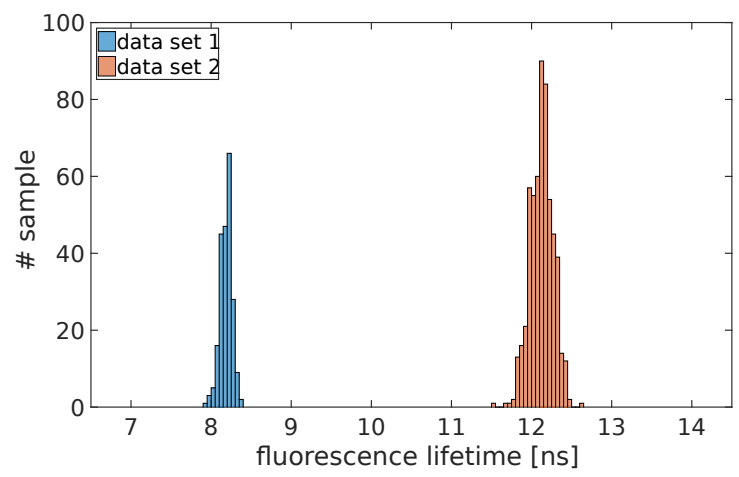

(b) Fluorescence lifetime distribution for data sets 1 and 2, where 10 individual droplet histograms were summed up to one input histogram.

Fig. 8: Distribution of the fluorescence lifetimes that were computed by the presented VHDL implementation. The input data are the measurements from [9] with additional postprocessing to remove the non-zero offset.

The original experimental data featured a non-zero offset that results in an overestimation of the fluorescence lifetime with the presented MLE algorithm and thus had to be removed before processing the histogram data. Further improvement will be to include an automated procedure for offset subtraction online during data acquisition.

For easier comparison with the results analysis from [9], the computed fluorescence lifetime values, which are computed with the same system setup described in Section 6, are plotted as histograms in Fig. 8a. Similar to the evaluation in Section 6, the parameters $\mu_{\tau}, \sigma_{\tau}$, the coefficient of variation and the F-value are listed in Table 7 , along with the reference values published in [9]. The results for $\sigma_{\tau}$, as well as the related coefficient of variation and the $\mathrm{F}$-value, are in good agreement with the reported values from [9]. The matching of these parameters indicates a high precision of the fluorescence lifetime estimate by the presented computation system.

However, the computed fluorescence lifetimes do not accurately match. The main reason for this is that in [9], the fluorescence lifetime of each droplet was computed offline with a 5-parameter fit implemented via a nonlinear least-square minimizing Matlab routine to fit each individual histogram, whereas here we perform a singleparameter fit based on the above MLE algorithm. In principle, both fitting procedure must give identical results in the case of purely monoexponential decays with zero offset, and strictly poissoninan noise distribution. Here, the original experimental data do feature a nonzero offset, which strongly influences the fluorescence lifetime estimate if it is not taken into account. Hence, for the present validation, we had to estimate and subtract this average offset before processing the histogram data, which reduces the discrepancy between the average lifetimes to less than $20 \%$ between both analysis schemes, as shown in Tables 7 and 8. In addition, the fluorescence decays are not rigorously monoexponential as can be readily seen for instance in Figure 7a (see residual systematic glitch at $15 \mathrm{~ns}$, induced by a slight defect in the FPGA-based TDC). We conclude that the 
average fluorescence lifetime extracted by both fitting procedure is not affected in the same way by the deviations from purely monoexponential decays.

Future improvements to enhance the accuracy of online fluorescence lifetime determination by the present MLE routine will be to include an automated procedure for offset subtraction during data acquisition, and to correct the residuals defects of the TDC.

Most importantly, while we just discussed the origins of inaccuracy (i.e. bias in the average lifetimes) we now underline the very good precision (width of the lifetime distributions) of the present method, which is similar to that obtained by the off-line 5-parameter fit of ref [9]. This very good precision is certainly the most critical and important result the present work, even in the case of non perfect data sets, because the precision is the most important criterion in screening applications where the goal is to be able to distinguish between two distinct values.

Finally, following the example in [9], the droplet histograms were summed up 10 by 10 to simulate a 10 -fold increase of the effective droplets exposure time during the measurement and reduce the Poisson noise by a factor of $\sqrt{10}$. The computed fluorescence lifetimes for these two newly created data sets are plotted in Fig. 8b and the results of the statistical analysis are listed in Table 8 together with the reference values from [9]. As expected, the precision, estimated by the $\sigma_{\tau}$ parameter, improves by a factor of $\sim \sqrt{10}$.

The measurement results in Tables 7 and 8 show that, remarkably, the presented MLE-based fluorescence lifetime computation system still produces very high precision results, even if the accuracy may be affected by imperfect data quality.

\section{Conclusion}

Time-resolved fluorescence analysis is among the primary research tools in biochemistry and biophysics [11]. Increasing the throughput of FADS systems improves the efficiency of drug discovery and can help finding new cures for diseases. This paper presents the implementation of a MLE based fluorescence measurement system. The results show that the algorithm offers superior fluorescence lifetime estimations, even for very low signal-to-noise inputs, i.e. low photon count. This enables shorter measurement times, which in turn increases the throughput of FADS systems, while also improving the measurement quality.

The MLE algorithm features indeed a higher computational complexity than non fitting algorithms. However, this paper shows that a flexible low cost
FPGA implementation is capable of processing high data rates in real time.

\section{References}

1. DE0-Nano-SoC User Manual. 9F., No.176, Sec.2, Gongdao 5th Rd, East Dist, Hsinchu City, 30070. Taiwan (2015). URL http://www.terasic.com.tw/attachment/ archive/941/DE0-Nano-SoC \_User \_manual.pdf

2. TimeHarp 260. Rudower Chaussee 29, 12489 Berlin, Germany (2016). URL http://www.picoquant.com/images/ uploads/downloads/timeharp260.pdf

3. Baret, J.C., Miller, O.J., Taly, V., Ryckelynck, M., ElHarrak, A., Frenz, L., Rick, C., Samuels, M.L., Hutchison, J.B., Agresti, J.J., Link, D.R., Weitz, D.A., Griffiths, A.D.: Fluorescence-activated droplet sorting (fads): efficient microfluidic cell sorting based on enzymatic activity. Lab Chip 9, 1850-1858 (2009). DOI 10.1039/B902504A. URL http://dx.doi.org/10.1039/B902504A

4. Digman, M.A., Caiolfa, V.R., Zamai, M., Gratton, E.: The phasor approach to fluorescence lifetime imaging analysis. Biophys J 94(2), L14-L16 (2008). DOI 10.1529/biophysj.107.120154. URL http://www.ncbi. nlm.nih.gov/pmc/articles/PMC2157251/. 120154[PII]

5. Franch, N., Alonso, O., Canals, J., Vilà, A., Herms, A., Dieguez, A.: A low cost fluorescence lifetime measurement system based on spad detectors and fpga processing. In: 2016 Conference on Design of Circuits and Integrated Systems (DCIS), pp. 1-6 (2016). DOI 10.1109/DCIS.2016.7845266

6. Giles, C.R., Burrus, C.A., DiGiovanni, D.J., Dutta, N.K., Raybon, G.: Characterization of erbium-doped fibers and application to modeling 980-nm and 1480-nm pumped amplifiers. IEEE Photonics Technology Letters 3(4), 363-365 (1991). DOI 10.1109/68.82113

7. Gorpas, D., Ma, D., Bec, J., Yankelevich, D.R., Marcu, L.: Real-time visualization of tissue surface biochemical features derived from fluorescence lifetime measurements. IEEE Transactions on Medical Imaging 35(8), 1802-1811 (2016). DOI 10.1109/TMI.2016.2530621

8. Hall, P., Selinger, B.: Better estimates of exponential decay parameters. The Journal of Physical Chemistry 85(20), 2941-2946 (1981). DOI 10.1021/j150620a019. URL http://dx.doi.org/10.1021/j150620a019

9. Ioanna Skilitsi, A., Turko, T., Cianfarani, D., Barre, S., Uhring, W., Hassiepen, U., Léonard, J.: Towards sensitive, high-throughput, biomolecular assays based on fluorescence lifetime. Methods and Applications in Fluorescence 5(3), 034,002 (2017). DOI 10.1088/2050-6120/ aa7f66

10. Köllner, M., Wolfrum, J.: How many photons are necessary for fluorescence-lifetime measurements? Chemical Physics Letters 200(1), 199 - 204 (1992). DOI http://dx.doi.org/10.1016/0009-2614(92)87068-Z. URL http://www.sciencedirect.com/science/article/pii/ $000926149287068 \mathrm{Z}$

11. Lakowicz, J.R.: Principles of Fluorescence Spectroscopy. third edn. Springer US (2006)

12. Léonard, J., Dumas, N., Causse, J.P., Maillot, S., Giannakopoulou, N., Barre, S., Uhring, W.: High-throughput time-correlated single photon counting. Lab Chip 14, 4338-4343 (2014). DOI 10.1039/C4LC00780H. URL http://dx.doi.org/10.1039/C4LC00780H

13. Li, D.D.U., Arlt, J., Tyndall, D., Walker, R., Richardson, J., Stoppa, D., Charbon, E., Henderson, R.K.: Video-rate 
fluorescence lifetime imaging camera with cmos singlephoton avalanche diode arrays and high-speed imaging algorithm. Journal of Biomedical Optics 16(9), 096,012096,012-12 (2011). DOI 10.1117/1.3625288. URL http: $/ /$ dx.doi.org/10.1117/1.3625288

14. Li, D.U., Arlt, J., Richardson, J., Walker, R., Buts, A., Stoppa, D., Charbon, E., Henderson, R.: Real-time fluorescence lifetime imaging system with a $32 \times 320.13 \mu \mathrm{m}$ cmos low dark-count single-photon avalanche diode array. Opt. Express 18(10), 10,257-10,269 (2010). DOI 10. 1364/OE.18.010257. URL http://www.opticsexpress. org/abstract. cfm?URI=oe-18-10-10257

15. Lieske, T., Uhring W. Dumas, N., Léonard, J., Fey, D.: Embedded fluorescence lifetime determination for high throughput real-time droplet sorting with microfluidics. In: 2017 Conference on Design and Architectures for Signal and Image Processing (DASIP) (2017)

16. Liu, J., Sun, Y., Qi, J., Marcu, L.: A novel method for fast and robust estimation of fluorescence decay dynamics using constrained least-squares deconvolution with laguerre expansion. Physics in Medicine and Biology 57(4), 843 (2012). URL http://stacks.iop.org/0031-9155/57/i= $4 / a=843$

17. Maus, M., Cotlet, M., Hofkens, J., Gensch, T., De Schryver, F.C., Schaffer, J., Seidel, C.A.M.: An experimental comparison of the maximum likelihood estimation and nonlinear least-squares fluorescence lifetime analysis of single molecules. Analytical Chemistry 73(9), 2078-2086 (2001). DOI 10.1021/ac000877g. URL http: //dx.doi.org/10.1021/ac000877g. PMID: 11354494

18. Paschotta, R., Nilsson, J., Tropper, A.C., Hanna, D.C.: Ytterbium-doped fiber amplifiers. IEEE Journal of Quantum Electronics 33(7), 1049-1056 (1997). DOI $10.1109 / 3.594865$

19. Rowley, M.I., Barber, P.R., Coolen, A.C.C., Vojnovic, B.: Bayesian analysis of fluorescence lifetime imaging data (2011). DOI 10.1117/12.873890. URL http://dx.doi. org/10.1117/12.873890

20. Schmid, L., Weitz, D.A., Franke, T.: Sorting drops and cells with acoustics: acoustic microfluidic fluorescenceactivated cell sorter. Lab Chip 14, 3710-3718 (2014). DOI 10.1039/C4LC00588K. URL http://dx.doi.org/ 10.1039/C4LC00588K

21. Swaminathan, R., Periasamy, N.: Analysis of fluorescence decay by the maximum entropy method: Influence of noise and analysis parameters on the width of the distribution of lifetimes. Proceedings of the Indian Academy of Sciences - Chemical Sciences 108(1), 39 (1996). DOI 10.1007/BF02872511. URL http://dx.doi. org/10.1007/BF02872511

22. Teh, S.Y., Lin, R., Hung, L.H., Lee, A.P.: Droplet microfluidics. Lab Chip 8, 198-220 (2008). DOI 10.1039/ B715524G. URL http://dx.doi.org/10.1039/B715524G

23. Tyndall, D., Rae, B.R., Li, D.D.U., Arlt, J., Johnston, A., Richardson, J.A., Henderson, R.K.: A high-throughput time-resolved mini-silicon photomultiplier with embedded fluorescence lifetime estimation in $0.13 \mu \mathrm{m}$ cmos. IEEE Transactions on Biomedical Circuits and Systems 6(6), 562-570 (2012). DOI 10.1109/TBCAS.2012. 2222639

24. Whitesides, G.M.: The origins and the future of microfluidics. Nature 442(7101), 368-373 (2006). DOI 10. 1038/nature05058. URL http://dx.doi.org/10.1038/ nature05058

25. Yu, H., Saleeb, R., Dalgarno, P., Li, D.D.U.: Estimation of fluorescence lifetimes via rotational invariance techniques. IEEE Transactions on Biomedical Engineering 63(6), 1292-1300 (2016). DOI 10.1109/TBME.2015. 2491364

26. Zhang, Z., Grattan, K.T.V., Hu, Y., Palmer, A.W., Meggitt, B.T.: Prony's method for exponential lifetime estimations in fluorescence-based thermometers. Review of Scientific Instruments 67(7), 2590-2594 (1996). DOI 10.1063/1.1147219. URL http://dx.doi.org/10.1063/ 1.1147219 


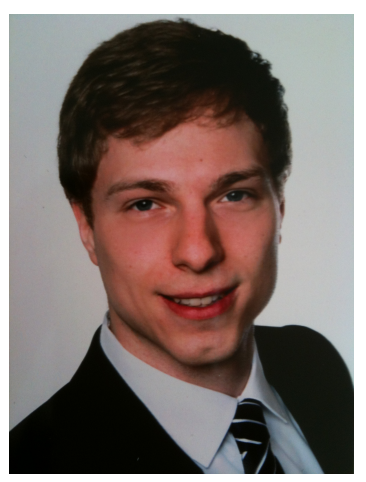

Tobias Lieske received his master degree in Information and Communication Technology at the Friedrich-AlexanderUniversity (FAU) ErlangenNürnberg in 2015. Currently he is a research fellow at FAU at the chair of computer architecture. There he focuses on low-power system design, architecture generation and accelerator design.

Wilfried Uhring re-

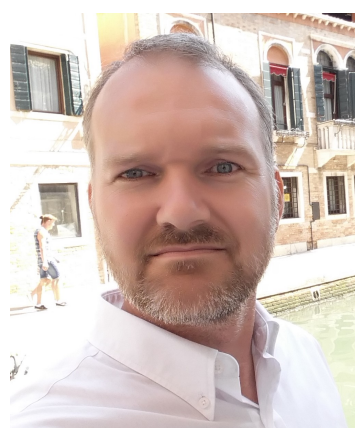
ceived the M.Sc degree in microelectronic and the Master's Degree in engineering physic in 1999 and the Ph.D. degree in optoelectronics from University of Strasbourg, France, in 2002. Since 1999, he worked on the field of high speed imaging. He designed several ultrafast optical detection devices such as streak camera and gated intensified camera with sub-nanoseconds to picoseconds resolution. Since 2003, he extend his research activity in integrated ultrafast optoelectronic CMOS devices such as solid-state streak camera and System on a Chip. In 2013, he joined the Engineering science, computer science and imaging laboratory (ICube) laboratory, university of Strasbourg and CNRS in France, where he managed the Heterogeneous System and Microsystem team (SMH) of the ICube laboratory.

Norbert Dumas re-

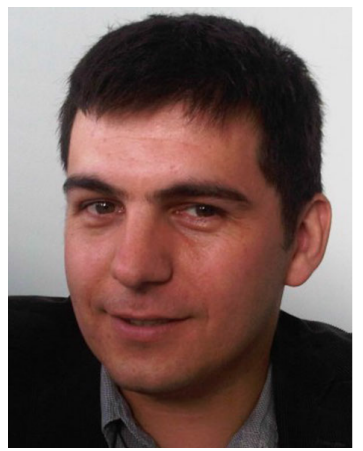
ceived his Ph.D. on MEMS design and test from University Montpellier II in 2005. He then worked for 2 years at University of Lancaster (UK) as a postdoc in the framework of a Network of Excellence. He was a research engineer at LIRMM/University Montpellier II. He joined the University of Strasbourg as an associate professor in 2012 and a member of the ICube Laboratory "Heterogeneous Systems and Microsystems" team. His research is mainly focussed on integrated sensors, actuators and microfluidics for lab on a chip.

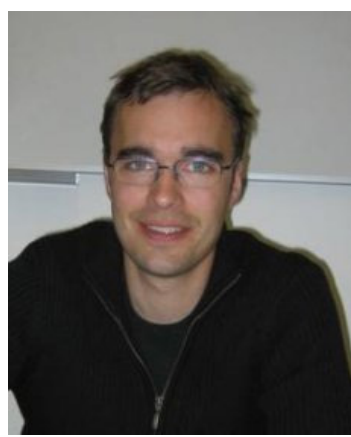

After an experimental PhD Work (2000-03, Laboratoire Kastler Brossel, Paris) and a Post-Doctoral fellowship (2004, Universiteit van Amsterdam) in quantum physics on ultracold gases, Jérémie Léonard was appointed as a CNRS researcher in 2005 at IPCMS (Strasbourg, France). Since then he has been using UV-Vis femtosecond spectroscopy to study ultrafast photoreactions of organic molecules in condensed phase. Among other research interests, he contributes the application of time-resolved fluorescence to the investigation of biomolecular interactions with both fundamental and applied targets.

Prof. Dr.-Ing. Diet-

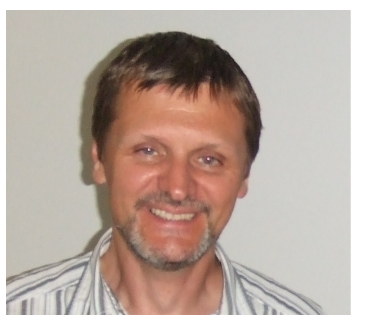
mar Fey holds a diploma degree in Computer Science from Friedrich-AlexanderUniversity (FAU) ErlangenNürnberg, Germany. In 1992 he received a Ph.D. from FAU with a work on an investigation about Using Optics in Computer Architectures. From 1994 to 1999 he researched at Friedrich-Schiller-University Jena where he made his habilitation. From 1999 to 2001 he worked as lecturer at University Siegen before he became a Professor for Computer Engineering at University Jena. Since 2009 he leads the Chair for Computer Architectureat Friedrich-Alexander-University ErlangenNürnberg (FAU). His research interests are in parallel computer architectures, parallel programming environments, parallel embedded systems, and memristive computing. 